\title{
Femtosecond laser fabrication of phase-shifted Bragg grating waveguides in fused silica
}

\author{
Jason R. Grenier, ${ }^{1, *}$ Luís A. Fernandes, ${ }^{1,2}$ J. Stewart Aitchison, ${ }^{1}$ Paulo V. S. Marques, ${ }^{2}$ and Peter R. Herman ${ }^{1}$ \\ ${ }^{1}$ Institute for Optical Sciences and the Department of Electrical and Computer Engineering, University of Toronto, \\ 10 King's College Road, Toronto, Ontario M5S 3G4, Canada \\ ${ }^{2}$ INESC-Porto, Departamento de Física e Astronomia da Universidade do Porto, Rua do Campo Alegre 687, 4169-007 Porto, Portugal \\ *Corresponding author: j.grenier@utoronto.ca
}

Received March 22, 2012; accepted April 19, 2012;

posted April 30, 2012 (Doc. ID 164997); published June 7, 2012

Phase-shifted Bragg grating waveguides (PSBGWs) were formed in bulk fused silica glass by femtosecond laser direct writing to produce narrowband $(22 \pm 3)$ pm filters at $1550 \mathrm{~nm}$. Tunable $\pi$ and other phase shifts generated narrow passbands in controlled positions of the Bragg stopband, while the accurate placement of multiple cascaded phase-shift regions yielded a rectangular-shaped bandpass filter. A waveguide birefringence of $(7.5 \pm 0.3) \times 10^{-5}$ is inferred from the polarization-induced spectral shifting of the PSBGW narrowband filters. @ 2012 Optical Society of America

OCIS codes: $230.1480,050.5080,230.7370,230.3120,140.3390$.

Femtosecond laser direct writing in bulk transparent dielectric materials presents an attractive single-step technique for generating three-dimensional (3D) optical circuits that cannot be fabricated using traditional, lithography-based techniques. The ability to induce refractive index modifications with focused femtosecond laser irradiation to inscribe waveguides in bulk glass has attracted considerable interest in the fabrication of numerous integrated optical devices [1]. The use of burst trains of femtosecond pulses enabled the fabrication of Bragg grating waveguides (BGWs) [2], which opened new directions for spectral filtering and temperature, strain, and other sensing applications in bulk glass. In traditional Bragg gratings, the insertion of a phase shift is used to introduce Fabry-Perot resonator effects that open a narrow transmission peak in the Bragg stopband. Such narrow filters offer much higher resolution for applications such wavelength demultiplexing in multichannel lightwave systems [3], single-frequency operation in distributed feedback lasers [4] and all-optical temporal differentiator [5].

Phase shifts were first demonstrated into a distributed grating type waveguide by etching away selected grooves in a narrow central region of a uniform semiconductor grating []․ Phase shifts were subsequently extended to fiber Bragg gratings (FBGs) by locally modifying the refractive index at a specific region along the grating by UV postprocessing [7] or localized postheating []. Femtosecond laser point-by-point writing has also been adopted to inscribe second-order Bragg gratings in the core of a single-mode fiber (SMF) with laser post-trimming of a weak phase shift [9] and most recently extended to phaseshifted Bragg grating waveguide (PSBGW) formation in bulk glass [10]. Phase-shifted features have also been induced simultaneously during laser exposure through a custom-designed phase-shifted mask [11] or by shifting the fiber relative to a uniform phase mask by a fraction of the grating period during beam scanning [12]. In preliminary work, our group applied a femtosecond laser to fabricate weak, $5 \mathrm{~mm}$ long, first-order PSBGW [13], creating both the waveguide and the phase-shifted grating simultaneously in a single exposure step.
In this Letter, an improved method for femtosecond laser writing of PSBGW is presented that offers individual phase and amplitude control over each of the laser pulse bursts to precisely control the exposure timing, energy dose, duty cycle, and periodicity of each waveguide segment along the BGW. Experimental results for femtosecond laser-written first-order PSBGWs without the need for any postprocess laser phase trimming or restrictions to writing gratings inside a pre-existing waveguide core are reported. In this way, the technique opens new directions for high-resolution and tailored spectral design of BGW devices flexibly in 3D integrated optical circuits. The polarization-induced shifting of the sharp spectral resonances provided a precise measure of the femtosecond laser induced waveguide birefringence. The accurate placement of multiple arbitrary phase shifts along the BGW permitted the insertion of spectral defects within the stopband, resulting in the formation of a rectangularly shaped bandpass filter.

A femtosecond fiber laser (IMRA America; $\mu$ Jewel D-400-VR) of 300 fs pulse duration was frequency doubled to $522 \mathrm{~nm}$ and applied at $500 \mathrm{kHz}$ to produce BGWs, as shown in Fig. 1. An acousto-optic modulator (AOM; NEOS 23080-3-1.06-LTD) created laser burst trains to form a segmented waveguide consisting of an array of partially overlapping refractive index voxels, as reported in [2]. PSBGW fabrication was enabled by

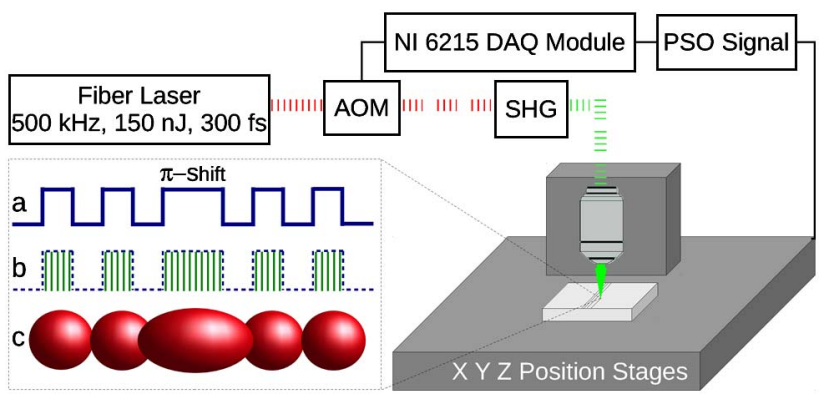

Fig. 1. (Color online) Femtosecond laser setup for the fabrication of PSBGWs. Inset, a, AOM modulation signal $(500 \mathrm{~Hz})$ with a $\pi$-phase-shift, $\mathrm{b}$, resulting laser bursts, and $\mathrm{c}$, refractive index voxels that produce the segmented waveguide. 
programming a data acquisition card (DAQ; NI 6215) to generate the AOM modulation signal such that the phase, amplitude, and duty cycle of each laser burst could be controlled. This flexible and complete control over the modulating signal enabled the insertion of an arbitrary number of phase shifts, whose position along the waveguide is controlled by using the position synchronized output (PSO) signal from the motion control stages (Aerotech ABL1000 with $2.5 \mathrm{~nm}$ resolution and $50 \mathrm{~nm}$ repeatability) to trigger the DAQ. Following previously developed formulas [2], a $500 \mathrm{~Hz}$ square-wave electrical signal with a $60 \%$ duty cycle, optimized to balance the trade-off of lower propagation losses and higher grating strength, was used to generate laser burst trains consisting of 600 pulses of $150 \mathrm{~nJ}$ energy. The laser burst trains were focused $75 \mu \mathrm{m}$ below the surface of a fused silica substrate (Corning 7980;50.8 mm $\times 25.4 \mathrm{~mm} \times 1 \mathrm{~mm}$ with all faces optically polished) with a $40 \times, 0.55 \mathrm{NA}$ aspherical lens, which produced a spot radius of $0.8 \mu \mathrm{m}\left(1 / e^{2}\right.$ intensity). The motion control stages translated the substrate with respect to the laser beam, which was linearly polarized parallel to the direction of motion, at a speed of $0.268 \mathrm{~mm} / \mathrm{s}$. The resulting first-order BGW had a Bragg resonance wavelength of $\lambda_{B}=1550 \mathrm{~nm}$ according to the Bragg relation: $\lambda_{B}=2 n_{\text {eff }} \Lambda$, where $n_{\text {eff }}$ is the effective in$\operatorname{dex}(1.445)$ of the waveguide mode and $\Lambda$ is the grating period $(536 \mathrm{~nm})$, which was controlled by the ratio of the scan speed to the AOM modulation frequency. All PSBGWs were $15 \mathrm{~mm}$ in length with $5 \mathrm{~mm}$ of unmodulated waveguide segments on either side for a total length of $25 \mathrm{~mm}$.

To characterize the PSBGWs, polarized transmission and reflection spectra were recorded by coupling light from a tunable laser (Photonetics Tunics-BT, 1 pm resolution) to a fiber circulator and end coupled into the device under test using SMFs. Index-matching oil was used at all glass-fiber interfaces to reduce Fresnel reflections and Fabry-Perot effects. Polarization control paddles and an in-fiber polarizer were used to manipulate the polarization to be horizontal or vertical for electric fields polarized parallel or perpendicular to the sample surface, respectively. All spectra were normalized relative to a direct fiber-to-fiber, oil-matched insertion loss.

The spectral response of the narrowband filters was controlled by specifying the location and amount of the phase shift to introduce in the grating periodicity. Figure 2 shows the transmission spectra for gratings with designed phase-shift values of $0, \pi, 3 \pi / 2$, and $\pi / 2$ located in the center of the grating. The precise centering of the $\pi$ (quarter-wave) phase defect as shown in the spectrum of Fig. 2(b) arises from an anticipated $268 \mathrm{~nm}$ offset added to the center two voxels of the grating by this technique. The $\pi$-shifted narrow filter was highly resolvable in both the reflection and transmission spectra when recorded separately for either horizontal (not shown) or vertical polarization [Fig. 2(b)], but it was otherwise unresolvable when probed with unpolarized light due to the waveguide birefringence. This defect line offers a contrast of at least $15 \mathrm{~dB}$ in reflection and $6 \mathrm{~dB}$ in transmission. By measuring the polarization-induced spectral shift of $(80 \pm 3) \mathrm{pm}$ between the narrow central transmission defect bands, as observed with vertical and horizontal polarization in Fig. $\underline{3}$, a birefringence of $\Delta n_{B}=(7.5 \pm 0.3) \times 10^{-5}$ was
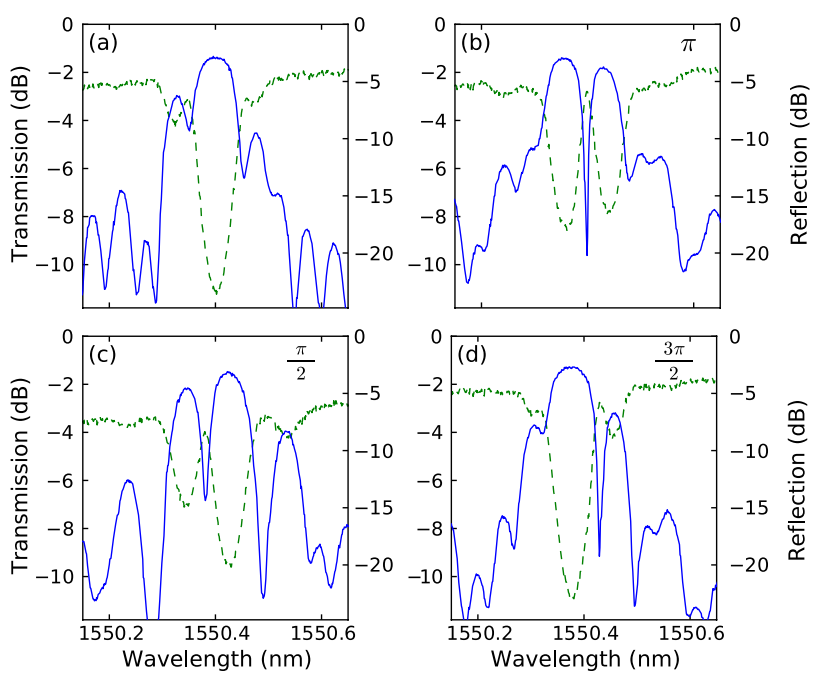

Fig. 2. (Color online) Vertically polarized transmission (dashed green curve) and reflection (solid blue curve) spectra for a BGW with (a) no phase shift, (b) $\pi$ phase shift, (c) $\pi / 2$ phase shift, and (d) $3 \pi / 2$ phase shift.

inferred for the present BGW devices. This birefringence, which can be due to asymmetric stresses induced by the femtosecond laser-writing process [14], has previously been harnessed to make integrated waveguide retarders [15] and polarization beam splitters [16]. The increased precision in the birefringence measurement offered by the PSBGW could be used in the design of these devices, or to assist with the tuning of laser conditions to form nonbirefringent waveguide devices, as discussed in [16]. The $3 \mathrm{~dB}$ bandwidth of the narrow transmission band of the $\pi$-PSBGW was measured to be $(22 \pm 3) \mathrm{pm}$, as shown in the inset in Fig. 3, producing a quality factor (Q) of 70,455, which is seven times higher than the $3 \mathrm{~dB}$ bandwidth of a BGW with no phase shift [Fig. 2(a)]. In this way, the birefringence reported above for the present PSBGW is sevenfold more precise than values previously available from BGWs $[15,16]$. Further, the narrow transmission bands reported here ( $22 \mathrm{pm})$ compare well to the $30 \mathrm{pm}$ width obtained by phase mask scanning [12] or $10 \mathrm{pm}$ width obtained by a phase-shifted phase mask [11].

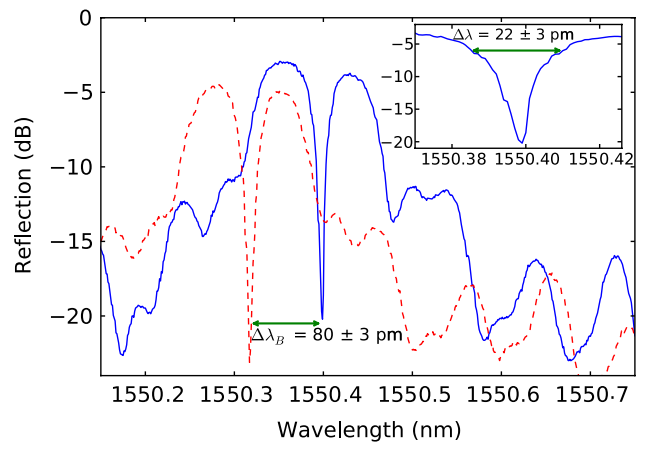

Fig. 3. (Color online) Vertical (solid blue curve) and horizontal (dashed red curve) polarized reflection spectra for a BGW with a single $\pi$ phase shift in the center of the grating. Inset, closeup of the vertical polarized reflection spectrum showing the $3 \mathrm{~dB}$ bandwidth. 


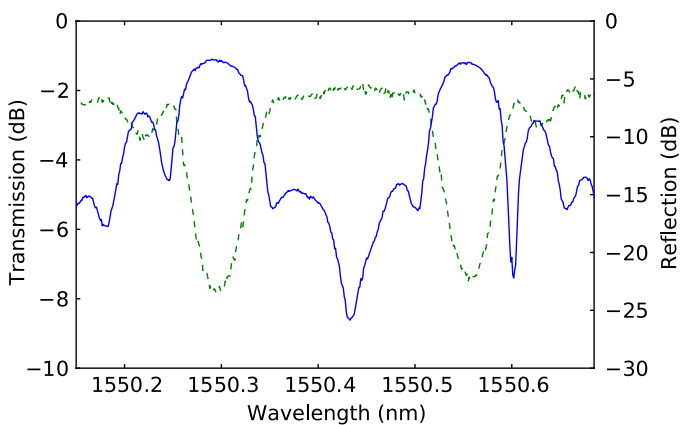

Fig. 4. (Color online) Vertically polarized transmission (dashed green curve) and reflection (solid blue curve) spectra of a BGW with five cascaded $\pi$ phase shifts.

The mode field diameters (MFDs) of the PSBGWs were measured to be $8.5 \mu \mathrm{m} \times 9.4 \mu \mathrm{m}$ at $1560 \mathrm{~nm}$, which closely matches the circular MFD of a SMF $(10.4 \mu \mathrm{m})$, yielding a very low modal mismatch loss of $0.11 \mathrm{~dB}$ per facet for fiber-to-waveguide coupling. The insertion losses are therefore mostly due to the waveguide propagation loss, found here to be $0.66 \mathrm{~dB} / \mathrm{cm}$, which is comparable to the $0.6 \mathrm{~dB} / \mathrm{cm}$ reported for the non-phase-shifted BGW [2]. Therefore, the phase-shift region does not introduce significant loss.

While PSBGWs offer spectrally narrow features that promise much improved resolution when used for sensing, many other applications require other spectral shapes that can now be constructed with a much higher resolution owing to the precise position and controllable phase-shift value that can be assigned to each grating voxel element formed by the laser. We follow the proposed design [17] for a rectangular passband in a FBG, extended here to direct laser writing, where six cascaded BGW sections having optimized length ratios of $1: 2: 2.17: 2.17: 2: 1$ and separated each by a $\pi$ phase shift. The resulting transmission and reflection spectra shown in Fig. $\underline{4}$ provide a passband ripple of $0.46 \mathrm{~dB}$ and a quality factor of 0.7 that compares well against the design values of $0.3 \mathrm{~dB}$ and 0.85 [17]. The rectangular passband in Fig. 4 offers a 3.5 times larger quality factor than the single $\pi$ PSBGW shown in Fig. 2(b).

Precise phase-shift control during burst laser writing of optical waveguides was shown to reproducibly generate narrow passbands in controlled positions of the Bragg stopband, opening new directions for high resolution and tailored spectral design of BGW devices in 3D integrated optical circuits. The present technique of controlling the phase, amplitude, and duty cycle of the laser burst trains will further enable apodized and chirped BGWs to be formed and the possibility for arbitrary tailoring of Bragg grating spectral shapes for pulse shaping [18]. These advantages can now be exploited for devices formed in bulk optics, extending beyond the limits of present-day fiber grating devices.

This work was supported by the National Science and Engineering Research Council of Canada, the Canadian Institute for Photonic Innovation, and the Portuguese Fundação para a Ciência e Tecnologia.

\section{References}

1. R. Osellame, G. Cerullo, and R. Ramponi, Femtosecond Laser Micromachining (Springer-Verlag, 2012).

2. H. Zhang, S. Eaton, and P. Herman, Opt. Lett. 32, 2559 (2007).

3. G. Agrawal and S. Radic, IEEE Photon. Technol. Lett. 6, 995 (1994).

4. H. Haus and C. Shank, IEEE J. Quantum Electron. 12, 532 (1976).

5. N. K. Berger, B. Levit, B. Fischer, M. Kulishov, D. V. Plant, and J. Azaña, Opt. Express 15, 371 (2007).

6. R. Alferness, C. Joyner, M. Divino, M. Martyak, and L. Buhl, Appl. Phys. Lett. 49, 125 (1986).

7. J. Canning and M. Sceats, Electron. Lett. 30, 1344 (1994).

8. D. Uttamchandani and A. Othonos, Opt. Commun. 127, 200 (1996).

9. G. D. Marshall, R. J. Williams, N. Jovanovic, M. J. Steel, and M. J. Withford, Opt. Express 18, 19844 (2010).

10. M. Ams, P. Dekker, G. Marshall, and M. Withford, Opt. Lett. 37, 993 (2012).

11. R. Kashyap, P. Mckee, and D. Armes, Electron. Lett. 30, 1977 (1994).

12. W. Loh, M. Cole, M. Zervas, S. Barcelos, and R. Laming, Opt. Lett. 20, 2051 (1995).

13. L. Fernandes, J. Grenier, P. Herman, J. Aitchison, and P. Marques, in Femtosecond Laser Microfabrication, OSA Technical Digest (CD) (Optical Society of America, 2009), paper LMTuC5.

14. V. Bhardwaj, P. Corkum, D. Rayner, C. Hnatovsky, E. Simova, and R. Taylor, Opt. Lett. 29, 1312 (2004).

15. L. Fernandes, J. Grenier, P. Herman, J. Aitchison, and P. Marques, Opt. Express 19, 18294 (2011).

16. L. Fernandes, J. Grenier, P. Herman, J. Aitchison, and P. Marques, Opt. Express 19, 11992 (2011).

17. R. Zengerle and O. Leminger, J. Lightwave Technol. 13, 2354 (1995).

18. K. Dolgaleva, A. Malacarne, P. Tannouri, L. Fernandes, J. Grenier, J. Aitchison, J. Azaña, R. Morandotti, P. Herman, and P. Marques, Opt. Lett. 36, 4416 (2011). 